\title{
Dielectric elastomers of interpenetrating networks
}

\section{Citation}

Suo, Zhigang, and Jian Zhu. 2009. “Dielectric Elastomers of Interpenetrating Networks." Applied Physics Letters 95 (23): 232909. https://doi.org/10.1063/1.3272685.

\section{Permanent link}

http://nrs.harvard.edu/urn-3:HUL.InstRepos:41461292

\section{Terms of Use}

This article was downloaded from Harvard University's DASH repository, and is made available under the terms and conditions applicable to Other Posted Material, as set forth at http:// nrs.harvard.edu/urn-3:HUL.InstRepos:dash.current.terms-of-use\#LAA

\section{Share Your Story}

The Harvard community has made this article openly available.

Please share how this access benefits you. Submit a story.

Accessibility 


\title{
Dielectric elastomers of interpenetrating networks
}

\author{
Zhigang Suo a) and Jian Zhu \\ School of Engineering and Applied Sciences, Harvard University, Cambridge, Massachusetts 02138, USA
}

(Received 13 October 2009; accepted 16 November 2009; published online 10 December 2009)

\begin{abstract}
Recent experiments have shown that a voltage can induce a large deformation in an elastomer of interpenetrating networks. To understand the observation, this paper describes a model of interpenetrating networks of long and short chains. As the voltage ramps up, the elastomer may undergo snap-through instability. The network with long chains fills the space and keeps elastomer complaint at small to modest deformation. The network with short chains acts as a safety net that restrains the elastomer from thinning excessively. (C) 2009 American Institute of Physics.
\end{abstract}

[doi:10.1063/1.3272685]

Subject to an electric field, a membrane of a dielectric elastomer reduces thickness and expands area. This mechanism of actuation is being developed for diverse applications, including soft robots, adaptive optics, and programmable haptic surfaces. ${ }^{1}$ Large deformation of actuation has been achieved in several ways, by using an elastomer with low modulus and high permittivity, ${ }^{2}$ by prestretching an elastomer, ${ }^{3-5}$ by spraying charge on an electrode-free elastomer, ${ }^{6}$ and by using an elastomer of interpenetrating networks. ${ }^{78}$ A fundamental understanding of these approaches is important to designing devices, and to discovering alternative ways to achieve large deformation of actuation.

The basic behavior of the dielectric elastomer is understood in terms of three quantities: the modulus $\mu$, the permittivity $\varepsilon$, and the electrical breakdown field $E_{B}$. The electric field needed to deform an elastomer appreciably is on the order $\sqrt{\mu / \varepsilon}$, namely $10^{8} \mathrm{~V} / \mathrm{m}$, assuming $\mu \sim 10^{5} \mathrm{~N} / \mathrm{m}^{2}$ and $\varepsilon \sim 10^{-11} \mathrm{~F} / \mathrm{m}$. This high field brings the elastomer near electrical breakdown, a mode of failure that may limit the deformation of actuation. For an elastomer with $\sqrt{\mu / \varepsilon} \ll E_{B}$, however, the deformation of actuation is often limited by another mode of failure, electromechanical instability. ${ }^{9}$

This paper focuses on interpenetrating networks (Fig. 1). In experiments, ${ }^{7,8}$ a membrane of network A swollen with monomers was held in a state of biaxial stretches, $\lambda_{1}^{\prime}$ and $\lambda_{2}^{\prime}$, while the monomers were cured to form network B. The interpenetrating networks had enhanced deformation of actuation, but the mechanism of this enhancement has been uncertain, and its full potential unknown. This paper describes a model of interpenetrating networks of long and short chains. The network with long chains fills the space and keeps the elastomer compliant at small to modest deformation. The network with short chains acts as a safety net that restrains the elastomer from thinning excessively.

The electromechanical instability has been analyzed by using a model outlined as follows. ${ }^{5,10-16}$ Let a membrane be in a state of stretches, $\lambda_{1}$ and $\lambda_{2}$ in the plane, and $\lambda_{3}$ through the thickness. The membrane is taken to be incompressible, $\lambda_{1} \lambda_{2} \lambda_{3}=1$, so that the elastic energy per unit volume is a function of the in-plane stretches, $W\left(\lambda_{1}, \lambda_{2}\right)$. For an incompressible solid in a state of stress, superposing a hydrostatic stress does not change the state of deformation. Conse-

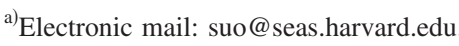

quently, the state of deformation caused by any state of stress can be represented by that caused by a state of biaxial stresses. We will use biaxial stresses $\sigma_{1}$ and $\sigma_{2}$ in the plane of the membrane, and set $\sigma_{3}=0$. The stresses are given by

$$
\begin{gathered}
\sigma_{1}=\lambda_{1} \partial W\left(\lambda_{1}, \lambda_{2}\right) / \partial \lambda_{1}-\varepsilon E^{2}, \\
\sigma_{2}=\lambda_{2} \partial W\left(\lambda_{1}, \lambda_{2}\right) / \partial \lambda_{2}-\varepsilon E^{2},
\end{gathered}
$$

where $E$ is the electric field.

We now analyze a membrane of interpenetrating networks. The state of the membrane in Fig. 1(a) is taken to be the state of reference. Network B is cured when the membrane is stretched to $\lambda_{1}^{\prime}$ and $\lambda_{2}^{\prime}$, called the mismatch stretches, Fig. 1(b). When the membrane is stretched to $\lambda_{1}$ and $\lambda_{2}$, Fig. 1(c), network A is stretched relative to the state of reference by $\lambda_{1}^{A}=\lambda_{1}$ and $\lambda_{2}^{A}=\lambda_{2}$, and network $B$ is stretched relative to the state in Fig. 1 (b) by $\lambda_{1}^{B}=\lambda_{1} / \lambda_{1}^{\prime}$ and $\lambda_{2}^{B}=\lambda_{2} / \lambda_{2}^{\prime}$. The two networks by themselves have the freeenergy functions $W^{A}\left(\lambda_{1}^{A}, \lambda_{2}^{A}\right)$ and $W^{B}\left(\lambda_{1}^{B}, \lambda_{2}^{B}\right)$. Let $\phi^{A}$ and $\phi^{B}$ be the volume fractions of the two networks in the membrane $\left(\phi^{A}+\phi^{B}=1\right)$. We neglect any chemical interaction between the two networks, and represent the free-energy function of the interpenetrating networks by

$$
W\left(\lambda_{1}, \lambda_{2}\right)=\phi^{A} W^{A}\left(\lambda_{1}^{A}, \lambda_{2}^{A}\right)+\phi^{B} W^{B}\left(\lambda_{1}^{B}, \lambda_{2}^{B}\right) .
$$

A polymeric molecule coils in many configurations due to thermal energy, and stiffens drastically when pulled near the full length of the molecule. To represent this stiffening effect, we adopt a model that represents the molecule by a freely jointed chain of links, ${ }^{17,18}$ and represents a network by

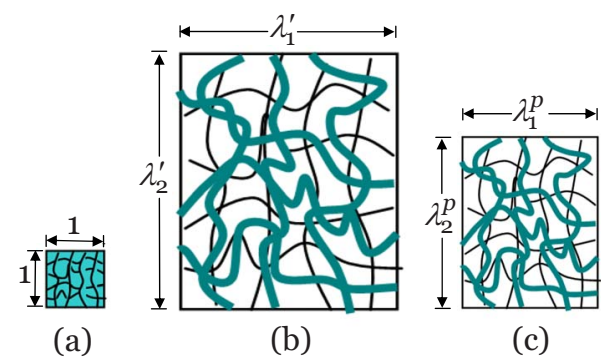

FIG. 1. (Color online) Plan view of a membrane. (a) Initially the membrane is network A swollen with monomers. (b) The membrane is then held in a state of biaxial stretches $\lambda_{1}^{\prime}$ and $\lambda_{2}^{\prime}$, while the monomers are cured to form network B. (c) After the external stresses are removed, the membrane contracts somewhat and preserves a state of stretches $\lambda_{1}^{p}$ and $\lambda_{2}^{p}$. In this preserved state, network $\mathrm{A}$ is in tension, and network $\mathrm{B}$ is in compression. 
the eight-chain model of Arruda and Boyce. ${ }^{19}$ The freeenergy function of the network is given in a parametric form

$$
\begin{aligned}
& W=\frac{k T}{v}\left(\frac{\zeta}{\tanh \zeta}-1+\log \frac{\zeta}{\sinh \zeta}\right), \\
& \Lambda=\sqrt{n}\left(\frac{1}{\tanh \zeta}-\frac{1}{\zeta}\right), \\
& \Lambda=\frac{1}{\sqrt{3}}\left(\lambda_{1}^{2}+\lambda_{2}^{2}+\lambda_{3}^{2}\right)^{1 / 2} .
\end{aligned}
$$

Here $k T$ is the temperature in the unit of energy, $v$ the volume per link, and $n$ the number of links in each chain. Equations (4)-(6) defines the function $W\left(\lambda_{1}, \lambda_{2}\right)$ using two intermediate parameters: the stretch $\Lambda$ and the normalized force $\zeta$ in each chain. In one limit, $\zeta \rightarrow \infty$, the chain approaches the limiting stretch, $\Lambda \rightarrow \sqrt{n}$. In the other limit, $\zeta \rightarrow 0$, the chain coils much below the limiting stretch, $\Lambda \ll \sqrt{n}$, and the model reduces to $W=(k T / 6 v) \zeta^{2}$ and $\Lambda=(\sqrt{n} / 3) \zeta$, which recovers the neo-Hookean model, with the small-strain shear modulus $\mu=k T /(v n)$.

We represent both networks by the above free-energy function, and differentiate the quantities for the two networks by superscripts $\mathrm{A}$ and $\mathrm{B}$. The stresses in the elastomer of interpenetrating networks are

$$
\begin{aligned}
\sigma_{1}= & \frac{\phi^{A} k T \zeta^{A}\left(\lambda_{1}^{2}-\lambda_{1}^{-2} \lambda_{2}^{-2}\right)}{3 v^{A} \sqrt{n^{A}} \Lambda^{A}} \\
& +\frac{\phi^{B} k T \zeta^{B}\left[\left(\lambda_{1}^{B}\right)^{2}-\left(\lambda_{1}^{B}\right)^{-2}\left(\lambda_{2}^{B}\right)^{-2}\right]}{3 v^{B} \sqrt{n^{B}} \Lambda^{B}}-\varepsilon E^{2}, \\
\sigma_{2}= & \frac{\phi^{A} k T \zeta^{A}\left(\lambda_{2}^{2}-\lambda_{2}^{-2} \lambda_{1}^{-2}\right)}{3 v^{A} \sqrt{n^{A}} \Lambda^{A}} \\
& +\frac{\phi^{B} k T \zeta^{B}\left[\left(\lambda_{2}^{B}\right)^{2}-\left(\lambda_{2}^{B}\right)^{-2}\left(\lambda_{1}^{B}\right)^{-2}\right]}{3 v^{B} \sqrt{n^{B}} \Lambda^{B}}-\varepsilon E^{2} .
\end{aligned}
$$

In reporting numerical results, we set $v^{A}=v^{B}=v$, and normalize the stresses by $k T / v$ and the electric field by $(k T / \varepsilon v)^{1 / 2}$. These factors take values $k T / v=4 \mathrm{MPa}$ and $(k T / \varepsilon v)^{1 / 2} \approx 3$ $\times 10^{8} \mathrm{~V} / \mathrm{m}$, assuming $T \approx 300 \mathrm{~K}, \varepsilon \approx 4 \times 10^{-11} \mathrm{~F} / \mathrm{m}$ and $v \approx 10^{-27} \mathrm{~m}^{3}$.

After the external stresses are removed, the elastomer preserves a state of stretches $\lambda_{1}^{p}$ and $\lambda_{2}^{p}$, which can be determined by setting $\sigma_{1}=\sigma_{2}=0$ and $E=0$ in Eqs. (7) and (8). In the neo-Hookean limit, we find the preserved stretch in direction 1

$$
\lambda_{1}^{p}=\frac{\left[\phi^{A} \mu^{A}+\phi^{B} \mu^{B}\left(\lambda_{1}^{\prime} \lambda_{2}^{\prime}\right)^{2}\right]^{1 / 6}\left[\phi^{A} \mu^{A}+\phi^{B} \mu^{B}\left(\lambda_{2}^{\prime}\right)^{-2}\right]^{1 / 6}}{\left[\phi^{A} \mu^{A}+\phi^{B} \mu^{B}\left(\lambda_{1}^{\prime}\right)^{-2}\right]^{1 / 3}} .
$$

The preserved stretch in direction 2 can be obtained by switching the subscripts 1 and 2. Figure 2 plots a numerical solution obtained from the Arruda-Boyce model, which agrees well with the neo-Hookean limit. This agreement is unsurprising because, in the preserved state, the end-to-end distances of chains are much below their full lengths.

External stresses can stretch the membrane relative to the preserved state. This stress-stretch relation can be deter-

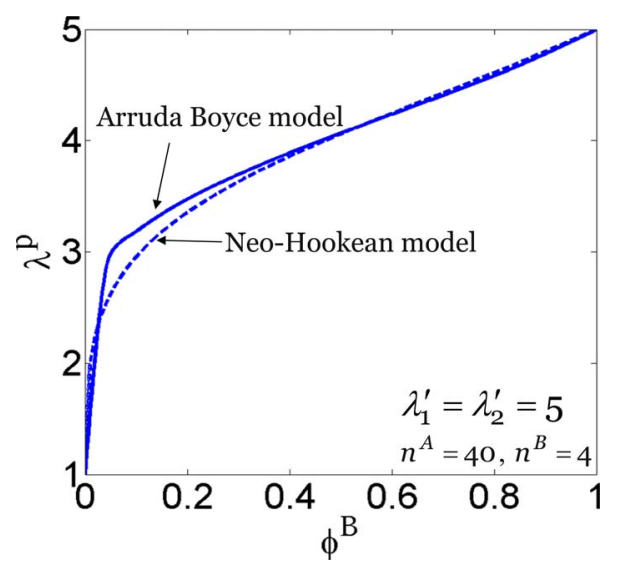

FIG. 2. (Color online) The preserved stretch as a function of the volume fraction of network $B$.

mined by setting $E=0$ in Eqs. (7) and (8), and is plotted in Fig. 3(a) for the equal-biaxial state, $\lambda_{1}=\lambda_{2}=\lambda$ and $\sigma_{1}=\sigma_{2}$ $=\sigma$. Note that the membrane with $\phi^{B}=0.4$ is stiffer than the membrane of single network B. This result is understood as follows. In the interpenetrating networks, chains of both networks are stressed, and are stiffer than the relaxed chains of the respective networks. This stress-induced stiffening compensates for the presence of the compliant network A.

Now consider an elastomer under no applied stress, $\sigma_{1}$ $=\sigma_{2}=0$, but is subject to a voltage. When the membrane stretches from the preserved state $\lambda_{1}^{p}=\lambda_{2}^{p}=\lambda^{p}$ to the state $\lambda_{1}=\lambda_{2}=\lambda$, the thickness of the membrane changes from $h^{p}$ to $h=\left(\lambda^{p} / \lambda\right)^{2} h^{p}$. Consequently, the voltage is given by $V=h E$ $=\left(\lambda^{p} / \lambda\right)^{2} h^{p} E$. This expression, together with Eq. (7), gives the voltage as a function of the stretch. In the neo-Hookean limit, this function can be obtained analytically
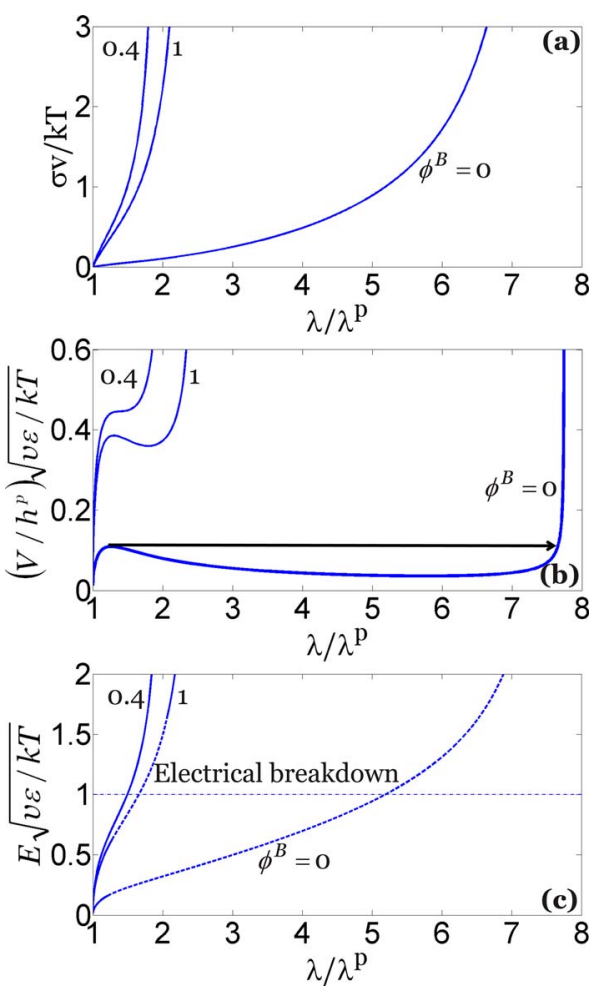

FIG. 3. (Color online) Stress (a), voltage (b), and electric field (c) as functions of the stretch relative to the state of preserved stretch $\left(\lambda^{\prime}=5, n^{A}=40\right.$, and $\left.n^{B}=4\right)$. 


$$
V(\lambda)=h^{p}\left(\lambda^{p} / \lambda\right)^{2} \varepsilon^{-1 / 2} \sqrt{\phi^{A} \mu^{A}\left(\lambda^{2}-\lambda^{-4}\right)+\phi^{B} \mu^{B}\left[\left(\lambda / \lambda^{\prime}\right)^{2}-\left(\lambda / \lambda^{\prime}\right)^{-4}\right]} .
$$

The function $V(\lambda)$ rises, reaches a peak at $\lambda / \lambda^{p}=2^{1 / 3}$, and then falls. When both networks are represented by the neoHookean model, the interpenetrating networks do not enhance the deformation of actuation compared to a membrane of single network.

For networks of finite chain lengths, the function $V(\lambda)$ is plotted in Fig. 3(b). The behavior is interpreted by tracing one curve, say the one labeled by $\phi^{B}=0$, as the stretch increases. The voltage rises, reaches a peak and falls, because the same voltage gives higher electric field when the membrane thins. After falling, the voltage rises again, because the chains approach the limiting stretches. The shape of the curve suggests coexistent states at a constant voltage, analogous to coexistent phases at a constant temperature. ${ }^{12}$ To simplify the discussion here, we will assume that the membrane is subject to a ramping voltage and, when the voltage reaches the peak, the membrane undergoes a snap-through instability, jumping from the peak to the state on the rising part of the curve; for example, see the arrow plotted for the curve $\phi^{B}=0$. Consequently, as the voltage ramps up, all the states between the peak and the state on the rising part of the curve are unreachable.

The snap-through instability itself does not cause failure, so long as the elastomer jumps to a state below the electrical breakdown field $E_{B}$. Figure 3(c) plots the electric field as a function of the stretch. The solid lines are states reachable when the voltage ramps up, and the dashed lines are states unreachable. Also plotted is a horizontal line representing the electrical breakdown field. In this example, the membrane of interpenetrating networks exhibits a larger stretch of actuation than either membrane of single network.

In plotting Fig. 3 , we have set $\lambda_{1}^{\prime}=\lambda_{2}^{\prime}=5, n^{A}=40$, and $n^{B}=4$. The choice of these parameters was guided by the experiments, ${ }^{7,8}$ which were guided by the notion that prestresses enhance deformation of actuation. Figure 4 plots the results by setting $\lambda_{1}^{\prime}=\lambda_{2}^{\prime}=1, n^{A}=500$, and $n^{B}=5$. By setting $\lambda_{1}^{\prime}=\lambda_{2}^{\prime}=1$, we have assumed that the interpenetrating networks are matched. After the external stresses are removed, both networks are relaxed, and the preserved stretches are $\lambda_{1}^{p}=\lambda_{2}^{p}=1$. This example illustrates a principle of operation that does not rely on prestresses. The network of long chains fills the space and keeps the elastomer compliant at small to modest stretches. The network of short chains acts as a safety net, restraining the elastomer from thinning down excessively.

In summary, snap-through instability may enable an elastomer to achieve a large deformation of actuation. This finding is not restricted to interpenetrating networks. The limited space here does not permit us to refine the form of the free energy. In particular, the network A in Fig. 1(a) and network B in Fig. 1(b) may not be regarded as fully relaxed. More refined models can relate molecular processes to macroscopic behavior, and aid the discovery of elastomers capable of even larger deformation of actuation.
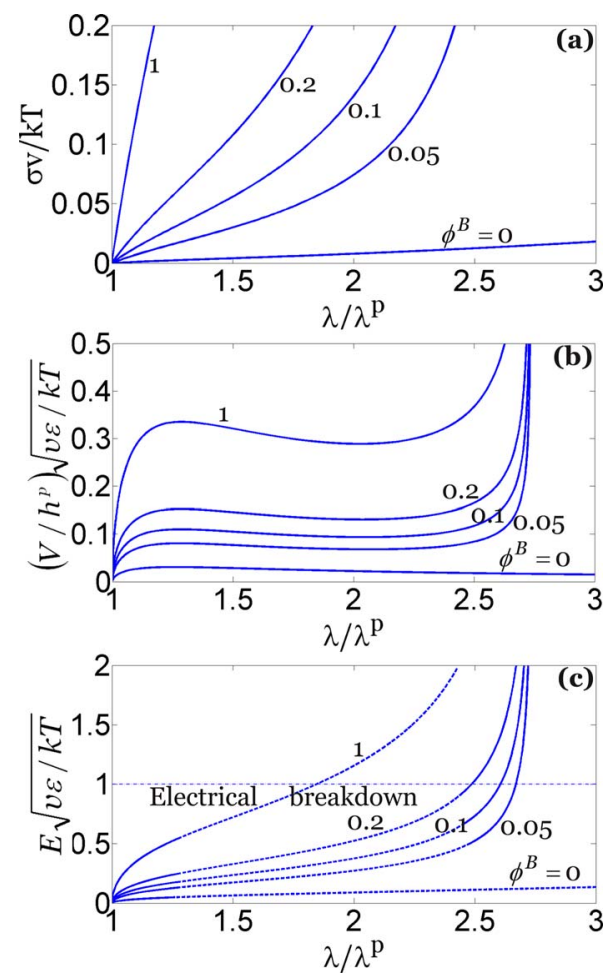

FIG. 4. (Color online) Stress (a), voltage (b), and electric field (c) as functions of the stretch relative to the state of preserved stretch $\left(\lambda^{\prime}=1, n^{A}\right.$ $=500$, and $n^{B}=5$ )

This work was supported by NSF through a grant on Soft Active Materials (Contract No. CMMI-0800161), and by DARPA through a contract on Programmable Matter (Contract No. W911NF-08-1-0143). Z.G.S. acknowledges the support of the Kavli Institute at Harvard University.

${ }^{1}$ F. Carpi, D. De Rossi, R. Kornbluh, R. Pelrine, and P. Sommer-Larsen, Dielectric Elastomers as Electromechanical Transducers (Elsevier, Oxford, 2008).

${ }^{2}$ R. Pelrine, R. Kornbluh, and J. Joseph, Sens. Actuators, A 64, 77 (1998).

${ }^{3}$ R. Pelrine, R. Kornbluh, Q. B. Pei, and J. Joseph, Science 287, 836 (2000)

${ }^{4}$ G. Kofod, P. Sommer-Larsen, R. Kronbluh, and R. Pelrine, J. Intell. Mater. Syst. Struct. 14, 787 (2003).

${ }^{5}$ J. S. Plante and S. Dubowsky, Int. J. Solids Struct. 43, 7727 (2006).

${ }^{6}$ C. Keplinger, M. Kaltenbrunner, N. Arnold, and S. Bauer (unpublished)

${ }^{7}$ S. M. Ha, W. Yuan, Q. B. Pei, and R. Pelrine, Adv. Mater. 18, 887 (2006).

${ }^{8}$ S. M. Ha, W. Yuan, Q. B. Pei, R. Pelrine, and S. Stanford, Smart Mater. Struct. 16, S280 (2007).

${ }^{9}$ K. H. Stark and C. G. Garton, Nature (London) 176, 1225 (1955).

${ }^{10}$ N. C. Goulbourne, E. M. Mockensturm, and M. Frecker, J. Appl. Mech. 72, 899 (2005).

${ }^{11}$ M. Wissler and E. Mazza, Sens. Actuators, A 138, 384 (2007)

${ }^{12}$ X. H. Zhao, W. Hong, and Z. G. Suo, Phys. Rev. B 76, 134113 (2007).

${ }^{13}$ X. H. Zhao and Z. G. Suo, Appl. Phys. Lett. 91, 061921 (2007).

${ }^{14}$ A. N. Norris, Appl. Phys. Lett. 92, 026101 (2008).

${ }^{15}$ R. Díaz-Calleja, E. Riande, and M. J. Sanchis, Appl. Phys. Lett. 93, 101902 (2008).

${ }^{16}$ Y. J. Liu, L. W. Liu, Z. Zhang, L. Shi, and J. S. Leng, Appl. Phys. Lett. 93, 106101 (2008).

${ }^{17}$ W. Kuhn and F. Grun, Kolloid-Z. 101, 248 (1942).

${ }^{18}$ L. R. G. Treloar, The Physics of Rubber Elasticity, 3rd ed. (Clarendon, Oxford, 1975)

${ }^{19}$ E. M. Arruda and M. C. Boyce, J. Mech. Phys. Solids 41, 389 (1993). 